\title{
Cecal Diverticulitis: an uncommon differential diagnosis from appendicitis
}

\section{Introduction}

Cecum diverticulosis is an uncommon condition in the Western world, its prevalence is based on several factors, such as nationality, culture and eating habits, with approximately $85 \%$ of the diverticula occurring in the descending and sigmoid colon. However, cecal diverticula present an incidence of more than $71 \%$ in Asian countries. They are divided into two types: the real ones, which have congenital origin, appearing during the sixth week of development, and the false ones, which do not involve the muscular layer. The progression of the cecal diverticulum is usually asymptomatic; however, symptoms can be found in $10-20 \%$ of patients according to complications such as inflammation, bleeding, perforation and bowel obstruction. In this context, the main complication of the cecal diverticulum is cecal diverticulitis, which was first described by Potier in 1912. Its signs and symptoms are very similar to those of appendicitis, such as abdominal pain in the right iliac fossa, nausea, vomiting, fever and leukocytosis, being the same the main differential diagnosis. In addition, it is important to note that the diagnosis of cecum diverticulitis is sometimes performed during the intraoperative appendectomy. Therefore, we will report the unusual case of a patient, who was submitted to surgery for suspected appendicitis and during the intraoperative period it was observed that he had diverticulitis of cecum

\section{Case report}

Patient, 42 years old, male, reported a lancinating pain in the lower right quadrant of the abdomen for 48 hours, associated with episodes of unmeasured fever and chills, denying vomiting, adynamia and changes in bowel habit. Physical examination revealed the presence of localized tenderness and painful decompression in right lower quadrant of abdomen, described as a Blumberg signal.

A hemogram showed 9,800 leukocytes per $\mathrm{mm}^{3}$. The patient had undergone a surgical procedure. It was initially performed as a Davis incision, which revealed hyperemia of the vermiform appendix and inflammatory mass surrounding a perforated cecum. So, the surgical team changed to xipho-pubic incision in order to perform a ileotiflectomy. The bowel transit was reconstructed by side-to-side ileotransverse anastomosis. The patient recovered well in the postoperative staying and was discharged after 6 days. Histopathological examination revealed: diverticular disease of the colon with diverticulitis and perforation associated with lymphoid hyperplasia of the cecal appendix.

\section{Discussion}

The solitary diverticulum of the cecum has a congenital origin, appearing during the sixth week of embryonic development. It is considered a true diverticulum because it is formed by all the layers of the wall of the intestine. This abnormality is usually asymptomatic and only manifests itself after an inflammation, hemorrhage or perforation. ${ }^{1-3}$

\author{
Volume 9 Issue 6 - 2018

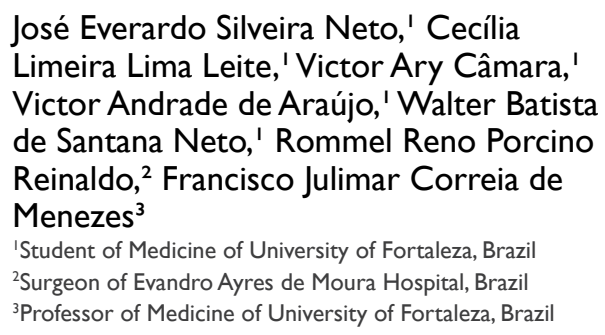

Correspondence: Francisco Julimar Correia de Menezes, Professor of Medicine of University of Fortaleza, B.P: 2300 Bento Albuquerque St. ap. 502 Lilac Tower - Cocó - Fortaleza - Ceará - Brazil, zip code 60.192.050 Tel + 5585 9861-7574, Email julimarnd@gmail.com

Received: May 12, 2018 | Published: November 20, 2018

Cecal diverticulitis is a rare cause of acute abdomen. The common presentation is a lower right quadrant abdominal pain and it is commonly misdiagnosed as acute appendicitis. Such mistake is responsible for more than $70 \%$ of cecum perforation in patients with cecum diverticulitis who had undergone a surgical procedure due to acute appendicitis preoperatively. Therefore, diverticulitis of the cecum is most often diagnosed intraoperatively. ${ }^{2,4}$

Some clinical manifestations can be used as conner stones that may help in suspicious case of cecal diverticulitis. Pain usually has a longer history and its onset is primarily in the right iliac fossa, where it remains. In acute appendicitis, the pain usually begins in the abdominal central region and then irradiated to the right iliac fossa. In addition, vomiting, nausea and anorexia are more common in acute appendicitis.

About $50 \%$ of the cecal diverticulum are found in the anterior cecal wall and can cause peritonitis. When the diverticulum is located in the posterior wall, it can generate great inflammation and simulate carcinoma. These diverticulum are usually approximately $2.5 \mathrm{~cm}$ from the ileocecal junction. ${ }^{5}$

Patients with abdominal pain in the right iliac fossa with probable diagnosis of appendicitis usually are not submitted to radiological examinations for confirmation. Computed tomography (CT) and Ultrasonography can be requested to differentiate these two diagnosis, with CT being more sensitive and more specific for the diagnosis of diverticulitis of the cecum than Ultrasonography. Thus, in case of suspected cecal diverticulitis in the preoperative period, the best examination to be asked for confirmation is Computed Tomography. $3,4,6$

The treatment of this disease is preferably surgical. However, if would be possible for the team perform the diagnosis before a surgical procedure and the disease is not complicated with perforation or 
peritonitis, it could be successfully treated with antibiotic. In cases where surgery is indicated for suspected acute appendicitis and cecal diverticulitis is diagnosed during surgery, treatment is controversial, and a incidental appendectomy and postoperative antibiotic therapy may be done. .,4, $^{4}$

There are four grades to characterize diverticulitis, depending on how the inflammation in the site is. Grade I refers to the inflamed diverticulum; grade II to inflammation in the cecum, grade III to the abscess and local fistula and grade IV to diffuse peritonitis and rupture or perforation of the abscess. If grade I treatment is appendectomy and diverticulectomy, if it is grade II, grade III or grade IV treatment is limited to ileocecostomy or right hemicolectomy. However, in cases of grade I, grade II and grade III, in which the diagnosis is made preoperatively, it may be considered conservative treatment. ${ }^{5,7}$

In conclusion, although cecal diverticulitis is a rare condition in Western countries, it should always be a differential diagnosis in cases of pain in the right iliac fossa, especially in cases of suspected acute appendicitis with unusual clinical features. Thus, in these cases, it is necessary to request complementary imaging tests to perform the correct diagnosis in the preoperative period and, in this way, to perform the most appropriate treatment according to the grade of diverticulitis.

\section{References}

1. Papapolychroniadis C, Kaimakis D, Fotiadis P, et al. Perforated diverticulum of the caecum. A difficult preoperative diagnosis. Report of 2 cases and review of the literature. Tech Coloproctol. 2004;8(Suppl 1):s116-s118.

2. Kyziridis DS, Parpoudi SN, Antoniou ND, et al. Cecal Diverticulitis is a Challenging Diagnosis: A Report of 3 Cases. Am J Case Rep. 2015;16:206-210.

3. Sahoo R, Kunar BM, padhi B, et al. Perforated solitary cecal diverticulum: An etiological challenge at emergency. Journal of Acute Medicine. 2016;6(2):49-51.

4. Karatepe $\mathrm{O}$, Gulcicek OB, Adas $\mathrm{G}$, et al. Cecal diverticulitis mimicking acute Appendicitis: a report of 4 cases. World J Emerg Surg. 20018;3:1619.

5. Koshy RM, Abusabeib A, Al-Mudares S, et al. Intraoperative diagnosis of solitary cecal diverticulum not requiring surgery: is appendectomy indicated?. World Journal of Emergency Surgery. 2016;11(1).

6. Salemis NS, Grapatsas K, Matzoukas I, et al. Perforated posterior cecal diverticulum: challenges in establishing an accurate preoperative diagnosis of a rare emergency. Am J Emerg Med. 2015;33(3):475.

7. Kalcan S, Başak F, Hasbahçeci M, et al. Intraoperative diagnosis of cecal diverticulitis during surgery for acute appendicitis: Case series. Ulus Cerrahi Derg. 2016;32(1):54-57. 